\title{
A High Energy X-ray Imager for Inertial Confinement Fusion at the National Ignition Facility
}

Riccardo Tommasini, Jeffrey A. Koch, Bruce Young, Ed Ng, Tom Phillips, Lucile Dauffy

May 7, 2006

High Temperature Plasma Diagnostics Williamsburg, VA, United States May 7, 2006 through May 11, 2006 
This document was prepared as an account of work sponsored by an agency of the United States Government. Neither the United States Government nor the University of California nor any of their employees, makes any warranty, express or implied, or assumes any legal liability or responsibility for the accuracy, completeness, or usefulness of any information, apparatus, product, or process disclosed, or represents that its use would not infringe privately owned rights. Reference herein to any specific commercial product, process, or service by trade name, trademark, manufacturer, or otherwise, does not necessarily constitute or imply its endorsement, recommendation, or favoring by the United States Government or the University of California. The views and opinions of authors expressed herein do not necessarily state or reflect those of the United States Government or the University of California, and shall not be used for advertising or product endorsement purposes. 


\title{
A HIGH ENERGY X-RAY IMAGER FOR INERTIAL CONFINEMENT FUSION AT THE NATIONAL IGNITION FACILITY*
}

\author{
Riccardo Tommasini, Jeffrey A. Koch, Bruce Young, Ed Ng, Tom Phillips, and \\ Lucile Dauffy \\ Lawrence Livermore National Laboratory, \\ P.O. Box 5508, Livermore, California 94550 USA
}

\begin{abstract}
X-ray imaging is a fundamental diagnostic tool for inertial confinement fusion (ICF) research, and provides data on the size and the shape of the core in implosions. We report on the feasibility and performance analysis of an ignition $\mathrm{x}$-ray imager to be used on cryogenic DT implosions at the National Ignition Facility. The system is intended to provide time-integrated, broadband, moderateenergy x-ray core images of imploding ICF capsules. It is optimized with respect to spatial-resolution, signal-to-background and signal-to-noise ratios, taking into account the extreme operating conditions expected at NIF due to high expected neutrons yields, gamma-rays, and $\mathrm{x}$-rays from laser-plasma interactions.
\end{abstract}

\section{Introduction}

$\mathrm{X}$-ray imaging is a fundamental diagnostic tool for ICF research. Indeed, providing data on the size and the shape of the core in implosions, it has a fundamental role in understanding the reasons for failures. Here we present the design and performance analysis of a HighEnergy X-Ray Imager (HEXRI) to be used on cryogenic DT implosions at NIF. The system HEXRI $^{(1)}$ will be pinhole-scintillator system, converting $\mathrm{X}$-rays to visible light and relaying the images to a distant detector. It will deliver time-integrated, broadband, $\mathrm{x}$-ray core images of imploding ICF capsules, in the $8 \mathrm{keV}-30 \mathrm{keV}$ energy range. Moreover, by crosscomparison of multiple differentially-filtered images it will have the ability to provide hotspot temperature and density maps and to observe areal density variations in the main fuel. Due to the unprecedented levels of backgrounds caused by neutrons, gamma-rays and x-rays generated by laser plasma interactions (LPI), the realization of such an imager is a difficult task. 


\section{System description}

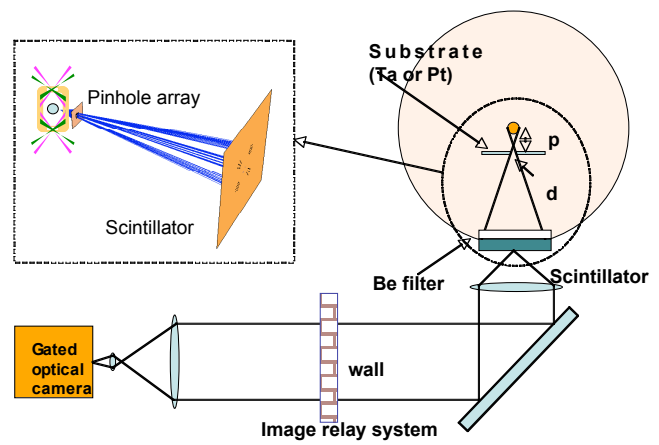

Figure 1: Simplified set up for the HEXRI.

Figure 1 shows a schematic of the instrument ${ }^{(1)}$. An array of pinholes mounted on the ignition cryogenic target positioner, close to target, at distance $\mathrm{p}$, will form highly magnified ( $100 \mathrm{x})$ images of the implosion cores onto a scintillator placed at the target chamber wall, about 5 meters form the source. Various filters placed in front of the scintillator will restrict the detected $\mathrm{x}$-ray spectrum. A telescope will relay the scintillator emission to a distant optical detector. Time gating will minimize the backgrounds while a mirror will help shielding against neutrons.

The spatial resolution, $\sigma$, at the object plane, is assumed to be the convolution of the geometrical resolution and the diffraction point spread function ${ }^{(2)}: \sigma^{2}=[d(1+1 / M)]^{2}+$ $(2.44 \lambda \mathrm{p} / \mathrm{d})^{2}$, where $\mathrm{M}$ is the magnification, $\mathrm{d}$ the pinhole diameter, and $\lambda$ the wavelength. The resolution has been optimized assuming the minimum possible pinholetarget distance: $50 \mathrm{~mm}$. Under these conditions a value of $5.8 \mu \mathrm{m}$ at $9 \mathrm{keV}$ is obtained for a pinhole diameter of $4 \mu \mathrm{m}$ and a magnification of $\sim 100 \mathrm{x}$. The primary sources of image blur in the whole cascaded system are radiation scattering in the scintillator and depth-offocus of the optical system. Both increase with the scintillator thickness, $t_{s}$. Figure 2 shows the percentage of variation of the resolution of the entire system vs. $t_{s}$, for value of 2 for the F-number. We note that the resolution is about $7 \mu \mathrm{m}$, at $9 \mathrm{keV}$, for a $200-$

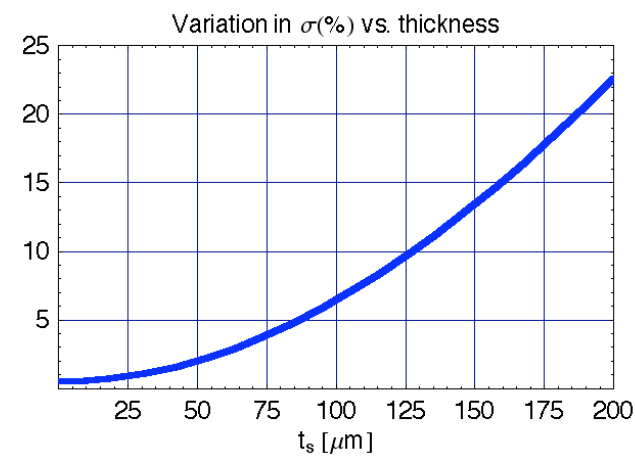

Figure 2: Variation of the resolution of the cascaded system (in \%) vs. scintillator thickness, for an F-number of 2 . $\mu \mathrm{m}-$ thick scintillator.

As anticipated in the introduction, the main pitfalls of the imaging system are the various sources of background. The emitted neutron distribution, peaking at 14 $\mathrm{MeV}$, requires detector time-gating to eliminate the late-arriving background, on times shorter than $50 \mathrm{~ns}$. This fixes an upper limit for the scintillator decay time. On the other hand the phosphor is required to have radiation hardness high enough to sustain tens of NIF shots without noticeable changes in detection properties. These two constraints appear to narrow our

choices to $\mathrm{BaF}$, LSO and GSO as scintillating materials.

The other sources of background are: core-emitted x-rays directly traversing the pinhole substrate (i.e. non-imaged); gamma rays from neutron-gamma reactions in the hohlraum and in the various objects inside the target chamber; x-rays from laser-plasma 
interactions (LPI). Neutron-gamma reactions will create a prompt background reducing the signal-to-background ratio (S/B) with increasing yield. LPI will produce prompt background up to hundreds of $\mathrm{keV} x$-rays that will reduce S/B independent of yield. Also in this case we can distinguish between non-imaged and imaged LPI x-rays, i.e. traversing the pinhole substrate and passing through the pinholes, respectively.

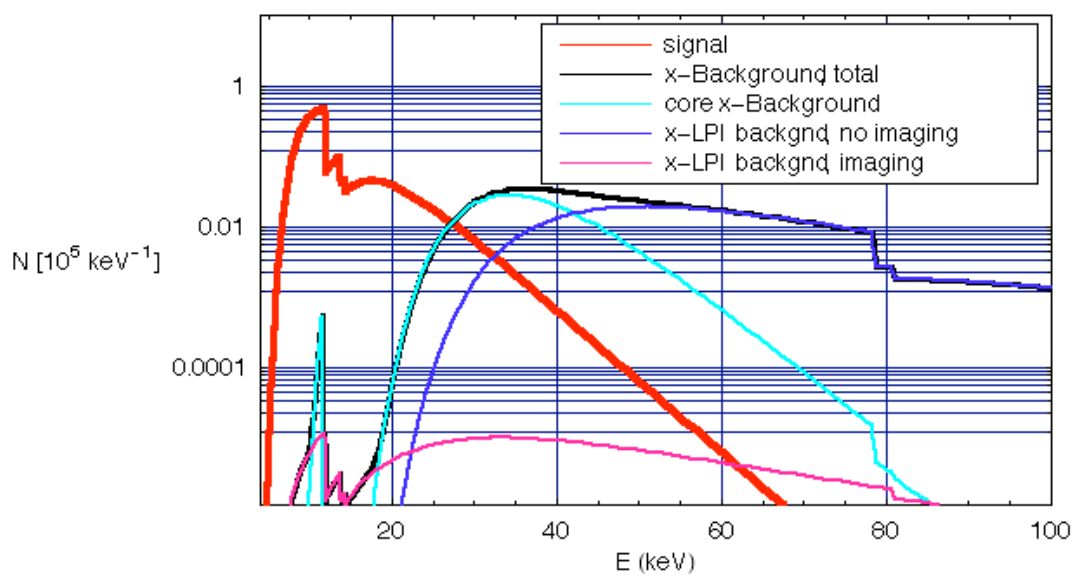

Figure 3: Spectra of the signal and the background $x$-rays incident on the scintillator. Pinhole-array substrates is $75 \mu \mathrm{m}$-thick Pt.

The expected $\mathrm{x}$-ray source brightness has been estimated from target design simulations $^{(3)}$ to be $10^{16} \mathrm{~W} / \mathrm{cm}^{2} \mathrm{Sr} \mathrm{keV}$ at $9 \mathrm{keV}$ (fizzle), with a duration of $100 \mathrm{ps}$. Figure 3 shows the spectra of the signal and the background $x$-rays incident on the scintillator. Calculations include the hohlraum structure and a 7-mm-thick Be vacuum window in front of the scintillator. The pinhole-array substrate is Pt, $75 \mu \mathrm{m}$-thick. The LPI background includes hot electron fluorescence from hohlraum wall but not from plasma. It has been evaluated by raytracing the photons from the various sources to the scintillator and simulating the transport into the scintillator using the EGS4 Monte Carlo $\operatorname{code}^{(4)}$, resulting in a S/B value of 3 for a $25 \mu \mathrm{m}$-thick GSO scintillator.

The spectrum from neutron-gamma reactions in various sources inside the NIF target chamber is modeled using the TART Monte Carlo code ${ }^{(5)}$. The results are shown in Figure 4. The photon transport into the scintillator is calculated using EGS4, including photoelectric, Compton, and pair-production effects. Assuming a neutron yiled of 1e17, the $\mathrm{S} / \mathrm{B}$ has the value of about 30 for a GSO scintillator, $25 \mu \mathrm{m}$ in thickness. Therefore, as far as neutron-gamma $\mathrm{S} / \mathrm{B}$ is of concern, the system can operate up to a yield of $\sim 1 \times 10^{18}$. Shielding would be required with a thicker scintillator or with additional neutrongammas sources. 
$\gamma$ per neutron/MeV into the sphere

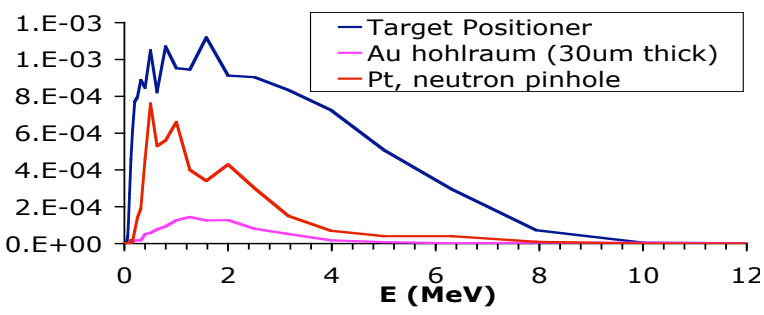

Figure 4: TART Monte Carlo simulation of the $\gamma$-ray spectrum incident onto the scintillator, for the main sources inside the NIF target chamber.
We note that the biggest source of background is represented by non-imaged LPI x-rays. However this contribution can be reduced by carefully shielding the regions out of the effective area of the pinhole array. This can be achieved using a snoutmounted pinhole array or, in the case of debris issues, by distributing the mass of the shielding material between the pinhole array

position and the detector, in form of multiple screens of increasing size. Assuming realistic conditions, our simulations show a reduction of the LPI x-ray background by a factor of more than 5 , resulting in a $\mathrm{S} / \mathrm{B}$ value of $\sim 7$.

The signal to noise ratio $(\mathrm{S} / \mathrm{N})$ has been calculated including the background and assuming the detection process in the scintillator to follow Poissonian statistics ${ }^{(6)}$. As a results, it has values higher than 10 , for a $25 \mu \mathrm{m}$-thick scintillator (GSO). The $\mathrm{S} / \mathrm{N}$ can be further improved by using a thinner Be window and a reflection coating on the front side of the scintillator (which would give an increase of a factor of $\sqrt{ } 2$ in $S / N$ ).

As said, the HEXRI's primary goal will be to record x-ray core emission images of pre-ignition cryogenic DT implosions to aid in recovery from possible failures to achieve ignition. Therefore the critical parameters to be measured are the core size and shape, using broadband imaging around $9-12 \mathrm{keV}$. Besides, HEXRI will provide hot-spot electron temperature and density, by cross-comparison of multiple energy (10-20 keV) differentially filtered $x$-ray core images, and main-fuel areal-density spatial variations by cross-comparison of multiple lower-energy $(<10 \mathrm{keV})$ differentially filtered x-ray core images that use core emission to backlight cold main-fuel plasma. Therefore the system will produce a 10x10 array of differentially filtered. The field of view (FOV) will be limited only by the detector size; e.g. a FOV of $100 \mu \mathrm{m}$ will require a scintillator with a transverse dimension of about $15 \mathrm{~cm}$. Given the scintillator area, the size of the gated optical camera will then determine the final magnification of the relay optical system.

\section{Conclusions}

Using a pinhole about $4 \mu \mathrm{m}$ in diameter a resolution varying between 8.5 and 4.5 $\mu \mathrm{m}$ in the $5-20 \mathrm{keV}$ spectral range is achieved $(5.8 \mu \mathrm{m}$ at $9 \mathrm{keV})$, limited by restrictions in the pinhole positioning. S/B ratios of about 3 have been estimated to be adequate for available scintillators showing fast enough decay time and suitable radiation hardness, namely GSO and LSO.

In particular, as far as neutron-gamma $\mathrm{S} / \mathrm{B}$ is of concern, a $25 \mu \mathrm{m}$ thick GSO scintillator can operate up to a yield of $\sim 1 \times 10^{18}$ without shielding. Background from LPI $\mathrm{X}$-rays appears to be more severe. However solutions exist to increase the S/B using shielding. Further reduction of background signals is possible by restricting the bandpass of the detection system.

The resulting $\mathrm{S} / \mathrm{N}$ is good: larger than 10 , for a $25 \mu \mathrm{m}$ thick GSO or LSO scintillator. 
Acknowledgments

This work was performed under the auspices of the U.S. Department of Energy by University of California, Lawrence Livermore National Laboratory under Contract W7405-Eng-48.

\section{References}

[1] R. Tommasini and J.A. Koch, UCRL-TR-211667, LLNL (2004).

[2] J. A. Koch et al., Appl. Opt. 37 (10) 1784 (1998).

[3] Steven Haan, LLNL, private communication.

[4] A.F. Bielajew et al., Stanford Linear Accelerator Center, Report SLAC-PUB-6499 (1994)

[5] E.F. Plechaty and R.E. Dye, UCID-17026, LLNL (1992)

[6] Swank, J. Appl. Phys. 44, 4199 (1973) 